\title{
OPTIMIZATION AND EVALUATION OF TOPICAL KETOCONAZOLE NANOEMULSION
}

\author{
ERNOVIYA ERNOVIYA ${ }^{1 *}$, MASFRIA MASFRIA ${ }^{1}$, KASMIRUL RAMLAN SINAGA²
}

${ }^{1}$ Department of Pharmaceutical Chemistry, Faculty of Pharmacy, University of Sumatera Utara, Medan, Indonesia. ${ }^{2}$ Department of Pharmaceutical Technology, Faculty of Pharmacy, University of Sumatera Utara, Medan, Indonesia. Email: ernoviya_28@yahoo.com

Received: 08 November 2017, Revised and Accepted: 23 January 2018

ABSTRACT

Objective: The present study is to formulate and optimization of surfactant, cosurfactant, and oil of ketoconazole nanoemulsion.

Methods: Ketoconazole was dissolved in surfactant, cosurfactant, and oil until saturated, clear solution extracted with methanol, its absorbance was measured using a UV spectrophotometer at $243 \mathrm{~nm}$. Comparisons of surfactants, cosurfactants, and oils are variously varied. Formulation method was performed using spontaneous nanoemulsion method.

Results: Surfactants, cosurfactants, and oils used for the nanoemulsion formula are tween 80, ethanol, and isopropyl myristate (IPM). Formulation of ketoconazole nanoemulsion with comparison of tween 80 concentration with ethanol (smix) 4:1 and smix with oil 9:1 was found that the formulae F1, F2, F3, F4, F5, F6, and F7 are unstable and the formulae F8, F9, F10, F11, and F12 are stable at the period of manufacture. The best physical stability tests from F8, F9, F10, F11, and F12 are F10.

Conclusions: Optimization of ketoconazole nanoemulsion formula was obtained at Tween 80, 36\% concentration, 9\% ethanol, and IPM 5\%.

Keywords: Optimization, Ketoconazole, Nanoemulsion.

(C) 2018 The Authors. Published by Innovare Academic Sciences Pvt Ltd. This is an open access article under the CC BY license (http://creativecommons. org/licenses/by/4. 0/) DOI: http://dx.doi.org/10.22159/ajpcr.2018.v11i5.23540

\section{INTRODUCTION}

Topical drug delivery methods have advantages over other delivery methods, one of which is to avoid the metabolism of the first pass effect on the liver [1]. Physical-chemical characteristic of drugs and excipients is taken into consideration in designing formulas to produce a product that is stable, efficacious, attractive, easy to formulate, and safe. These characteristics affect several factors: Particle size distribution, drug dissolution rate, bioavailability, uniformity of content, taste, texture, color, and stability. The particle size and solubility of the drug have an effect on the formulation because the drugs entering the blood circulation must be in solution form to produce the desired effect.

Ketoconazole is included in Class II of Biopharmaceutical Classification System means, including a class of low solubility and high permeability, the increased solubility of this drug is of concern to pharmaceutical researchers [2]. The absorption of ketoconazole orally is not maximal due to the solubility and the side effects it causes to overcome the deficiencies of this conventional system a new drug delivery system is required. Topical ketoconazole available on the market today, such as cream has side effects such as rash, itching, irritation, pain, and redness, therefore, to overcome this problem, requires a new drug delivery system such as nanoemulsion. Nanoemulsion has been widely used as a vehicle in topical medicine and is an alternative to insoluble, topical, or oral drugs.

Nanoemulsions are thermodynamically stable dispersions of two immiscible liquids (oil and water) which are stabilized using a surfactant and cosurfactant molecule. They may be either transparent or translucent and have a droplet size of 5-200 nm [3]. They are well tolerated orally on the skin and mucous membrane when used to deliver topically active drugs. Nowadays, increasing drug loading, enhancing drug solubility, and bioavailability are the most important advantages encouraging the usage of nanoemulsion as drug delivery carriers. A topical nanoemulsion is a form of delivery for a drug that is difficult to dissolve and has side effects when administered orally by increasing the penetration of the drug through the skin [4]. Nanoemulsions comprise safe surfactants with or without other emulsifiers to improve stability, oil (natural/synthetic/semi-synthetic) and cosurfactant [4].

The method of nanoemulsion formulation is divided into two methods that use high energy and low energy. High energy formulations require tools such as high-pressure homogenizers, microfluidizers, and sonicators, low energy formulation methods dependent on the solubility of the active substance so that it is more efficient to make a small droplet nanoemulsion. Nanoemulsions produced with low energy methods depend on the spontaneous formation of emulsions based on the phase behavior of certain surfactant, oil, and water systems. There is interest in using lower energy techniques in the emulsion formation process due to the economic benefits and increasing amounts of research have been conducted to investigate the utility of different lowenergy approaches. Self-emulsifying systems offer a strategy for dealing with the low bioavailability of compounds (drugs and oils) that are not easily dissolved in water [5].

A low energy emulsification or spontaneous emulsification method used by the laboratory scale to achieve small droplet size using simple instruments [6]. The advantage of the low energy method is that it can use simple equipment such as a magnetic stirrer, which includes low energy manufacturing methods are phase inversion temperature and phase inverse composition (PIC). The nanoemulsion method of PIC which is often performed for laboratory scale is by spontaneous emulsification $[7,8]$.

\section{MATERIALS AND METHODS}

\section{Materials}

Ketoconazole (PT. Kimia Farma Plant Tj Morawa Medan), coconut oil, castor oil, sesame oil, propylene glycol (PT Brataco, Indonesia) Tween 20 , Tween 80 , polyethylene glycol 400, isopropyl myristate (IPM), methanol, ethanol, butyl hydroxy toluene (Merck, Germany). 
Screening of ketoconazole solubility in oil surfactant and cosurfactant An excess amount of drug ( $\pm 250 \mathrm{mg}$ ) in $5 \mathrm{ml}$ of selected oils, surfactants, and cosurfactants (coconut oil, castor oil, sesame oil, polyethylene glycol 400, Tween 80, Tween 20, propylenglycol, glycerin, and ethanol) fixed by the vortex mixer. The mixture is stirred in the shaker for $24 \mathrm{~h}$. The ketoconazole sample was taken from the saturated oil phase and centrifuged at $3000 \mathrm{rpm} 15 \mathrm{~min}$. The solubility of drugs in oils, surfactants, and cosurfactants is determined by the supernatant layer of the centrifuged sample. Then $1 \mathrm{ml}$ of supernatant layer was diluted up to $10 \mathrm{ml}$ using methanol. The concentration of ketoconazole was then measured by a UV spectrophotometer at $243 \mathrm{~nm}$ [9-11].

Optimization of oils, surfactants, and cosurfactants

Optimization of surfactant concentrations, cosurfactant and oil were using different ratio The ratio of surfactant mixture to cosurfactant (smix) varies from $4: 1,3: 1,2: 1$, and $1: 1$. The ratio of oil and smix concentration is $1: 9,2: 8,3: 1,4: 6,5: 5,6: 4,7: 3,8: 2$, and 9:1. Aquades is added with titration and stirred using a magnetic stirrer until it becomes translucent and no phase separation [10].

\section{Formulation of nanoemulsion}

Nanoemulsion formulation was performed using spontaneous emulsification method. Ketoconazole is added to the oil phase which has added butylated hydroxytoluene to homogeneous, add a smix solution which is a mixture of surfactant and cosurfactant, then stirred with the magnetic stirrer until homogeneous. Aquades is added by means of titration, stirred continuously until nanoemulsion is formed which is marked by the formation of a translucent solution [10].

\section{Evaluation of nanoemulsion}

\section{Organoleptic}

Observations on any changes of color, odor, clarity, and phase separation were made.

\section{The $\mathrm{pH}$ value}

An important parameter of nanoemulsion evaluation is $\mathrm{pH}$ determination. The excipients used in the formulation decide the $\mathrm{pH}$ of the final preparation, and hence, the route of administration. The $\mathrm{pH}$ of the formulation was measured using a digital $\mathrm{pH}$ meter. The results were taken in triplicate to reduce the error [12].

\section{Viscosity}

The viscosity of nanoemulsion was measured using the Brookfield viscometer at room temperature $\left(23^{\circ} \mathrm{C} \pm 2^{\circ} \mathrm{C}\right)$. Viscosity measurements used two spindles speed and experiments were conducted 3 times [10].

\section{Nanoemulsion type}

Determination of nanoemulsion type was done by dissolving the emulsion in methylene blue. If the preparation is a type of oil in water, then the color will dissolve and diffuse. If the preparation is a type of water in oil, the methylene blue globules are located on the surface.

\section{Particle size and globule size distribution}

Particle size and globule size distribution were measured using particle size analyzer (PSA) SZ-100 [13].

\section{Physical stability test}

These tests included the cycling test, 8-week storage at temperatures of $4^{\circ} \mathrm{C} \pm 2^{\circ} \mathrm{C}, 25^{\circ} \mathrm{C} \pm 2^{\circ} \mathrm{C}$, and $40^{\circ} \mathrm{C} \pm 2^{\circ} \mathrm{C}$, and the centrifugation test speed of $3800 \mathrm{rpm}$ for $5 \mathrm{~h}$ [13].

\section{RESULT AND DISCUSSION}

\section{Solubility study}

The solubility of ketoconazole in oil, surfactant, and cosurfactant is shown in Table 1. From Table 1 result, the best solubility of ketoconazole is Tween 80 (surfactant), ethanol (cosurfactant), and IPM (oil).
Optimization of oils, surfactants, and cosurfactants

Mixing is done carefully and observed so that the unstable preparations which have cloudy and phase separation are not selected for further experiments. The aim of the optimization from each stage in the formulation is to get the best formula. The results from the observations of mixtures of tween 80 and ethanol (smix) with IPM are shown in Table 2.

The best comparison of tween 80 and ethanol (smix) is 4:1 which will be taken to make the nanoemulsion formula. The ratio of smix and oil is 9:1 and the ketoconazole nanoemulsion is made as shown in Table 3.

The excipient concentration of the F1, F2, F3, F4, and F5 nanoemulsions formula has not been able to stabilize the preparation because after stirring it is still cloudy and precipitating. Formulae F6 and F7 were initially stable and clear, but after waiting for some time, the formula became slightly cloudy and precipitated because the excipient concentration has not been able to stabilize nanoemulsion. Formulae F8 to F12 are stable and clear can be continued for further testing, while for formulae F1 to F7 cannot be continued because they were not stable.

\section{Evaluation of nanoemulsion}

The results of the evaluation of nanoemulsion in the $1^{\text {st }}$ week are shown in Table 4

\section{Organoleptic}

Formulae F8, F9, F10, F11, and F12 have a soft yellow color, slightly viscous, translucent, and homogeneous and have no phase separation. This indicates that the concentration of oil (IPM), surfactant (tween 80), and cosurfactant (ethanol 96\%) used is appropriate. When applied to the skin, the formula easily dissolves and leaves a slightly sticky nuance. This is because the concentration of tween 80 is high.

\section{pH}

The desired $\mathrm{pH}$ of the formulation is in the $\mathrm{pH}$ range of the skin (4.56.5). PH should not be too acid because it can irritate the skin should not be too alkaline because it can cause scaly skin [14]. All the formulas that are made have a $\mathrm{pH}$ that is still within the $\mathrm{pH}$ range of the skin.

Table 1: Solubility screening of ketoconazole

\begin{tabular}{ll}
\hline Excipients & Solubility $(\mathbf{m g} / \mathbf{m l})$ \\
\hline Tween 80 & 35.945 \\
Tween 20 & 16.733 \\
Propylene glycol & 12.486 \\
Polyethylene glycol 400 & 22.781 \\
Ethanol & 35.191 \\
Isopropyl myristate & 25.946 \\
Coconut oil & 5.946 \\
Castor oil & 10.691 \\
Sesame oil & 19.664 \\
\hline
\end{tabular}

Table 2: Observation of mixed comparisons of tween 80 and ethanol (smix) with IPM

\begin{tabular}{|c|c|c|c|c|}
\hline \multirow[t]{2}{*}{$\begin{array}{l}\text { Smix: } \\
\text { oil }\end{array}$} & \multicolumn{4}{|c|}{$\begin{array}{l}\text { Observations of mixtures of tween } 80 \text { and } \\
\text { ethanol (smix) }\end{array}$} \\
\hline & 1:1 & $2: 1$ & $3: 1$ & $4: 1$ \\
\hline $1: 9$ & $\sqrt{\sqrt{ }}$ & $\sqrt{\sqrt{ }}$ & $\sqrt{\sqrt{ }}$ & $\sqrt{\sqrt{ }}$ \\
\hline $2: 8$ & $\sqrt{ } \sqrt{ }$ & $\sqrt{\sqrt{ }}$ & $\sqrt{\sqrt{ }}$ & $\sqrt{\sqrt{ }}$ \\
\hline $3: 7$ & $\sqrt{ } \sqrt{ }$ & $\sqrt{\sqrt{ }}$ & $\sqrt{\sqrt{ }}$ & $\sqrt{\sqrt{ }}$ \\
\hline $4: 6$ & $\sqrt{ } \sqrt{ }$ & $\sqrt{\sqrt{ }}$ & $\sqrt{\sqrt{ }}$ & $\sqrt{ } \sqrt{ }$ \\
\hline $5: 5$ & $\sqrt{ } \sqrt{ }$ & $\sqrt{\sqrt{ }}$ & $\sqrt{\sqrt{ }}$ & $\sqrt{\sqrt{ }}$ \\
\hline $6: 4$ & $\sqrt{ } \sqrt{ }$ & $\sqrt{\sqrt{ }}$ & $\sqrt{\sqrt{ }}$ & $\sqrt{ } \sqrt{ }$ \\
\hline $7: 3$ & $\sqrt{\sqrt{ }}$ & $\sqrt{\sqrt{ }}$ & $\sqrt{\sqrt{ }}$ & $\sqrt{\sqrt{ }}$ \\
\hline $8: 2$ & $\sqrt{\sqrt{ }}$ & $\sqrt{\sqrt{ }}$ & $\sqrt{ } \sqrt{ }$ & $\sqrt{ } \sqrt{ }$ \\
\hline $9: 1$ & $\sqrt{ } \sqrt{ }$ & $\sqrt{\sqrt{ }}$ & $\sqrt{\sqrt{ }}$ & $\sqrt{ }$ \\
\hline
\end{tabular}

$\sqrt{\sqrt{ }}$ : Cloudy, $\sqrt{ } \sqrt{ }$ : Slightly cloudy, $\sqrt{ }$ : Translucent, IPM: Isopropyl myristate 
Table 3: Optimization of drug loaded nanoemulsion

\begin{tabular}{|c|c|c|c|c|c|c|c|c|c|c|c|c|}
\hline \multirow[t]{2}{*}{ Composition (\%) } & \multicolumn{12}{|c|}{ Formula } \\
\hline & F1 & F2 & F3 & F4 & F5 & F6 & F7 & F8 & F9 & F10 & F11 & F12 \\
\hline Ketoconazole & 2 & 2 & 2 & 2 & 2 & 2 & 2 & 2 & 2 & 2 & 2 & 2 \\
\hline IPM & 0.5 & 1.0 & 1.5 & 2.0 & 2.5 & 3.0 & 3.5 & 4.0 & 4.5 & 5.0 & 5.5 & 6.0 \\
\hline Ethanol & 0.9 & 1.8 & 2.7 & 3.6 & 4.5 & 5.4 & 6.3 & 7.2 & 8.1 & 9.0 & 9.9 & 10.8 \\
\hline Tween 80 & 3.6 & 7.2 & 10.8 & 14.4 & 18.0 & 21.6 & 25.2 & 28.8 & 32.4 & 36.0 & 39.6 & 43.2 \\
\hline BHT & 0.1 & 0.1 & 0.1 & 0.1 & 0.1 & 0.1 & 0.1 & 0.1 & 0.1 & 0.1 & 0.1 & 0.1 \\
\hline Aquades & 92.9 & 87.9 & 82.9 & 77.9 & 72.9 & 67.9 & 62.9 & 57.9 & 52.9 & 47.9 & 42.9 & 37.9 \\
\hline
\end{tabular}

IPM: Isopropyl myristate, BHT: Butylated hydroxytoluene

Table 4: Evaluation of nanoemulsion in the $1^{\text {st }}$ week

\begin{tabular}{llllll}
\hline Formula & Color & Odor & Clarity & pH & Viscosity \\
\hline F8 & Soft yellow & Specific & Translucent & 6.10 & 516.67 \\
F9 & Soft yellow & Specific & Translucent & 6.03 & 533.33 \\
F10 & Soft yellow & Specific & Translucent & 6.10 & 550.00 \\
F11 & Soft yellow & Specific & Translucent & 5.97 & 558.33 \\
F12 & Soft yellow & Specific & Translucent & 5.97 & 583.33 \\
\hline
\end{tabular}

\section{Viscosity}

Measurement of viscosity was performed using Brookfield viscometer. Five formulas of this emulsion showed various viscosity. Based on the optimal results in the increase of oil concentration in the formula, the concentration of tween 80 as surfactant will also increase so that it will affect its viscosity; in Fig. 1, it is shown that the high concentration of tween 80 will increase its viscosity. The higher concentration of tween 80 , it can decrease the globule size. Diminished globule size increases the number of particles, which is associated with the system's attempt to maintain a stable mass. Increasing the number of small globules will increase the interaction between globules, thus increasing the viscosity $[15,16]$.

\section{Nanoemulsion type}

All formulas contain nanoemulsion type oil in water $(\mathrm{o} / \mathrm{w})$. This is because most of the components present in the formula are hydrophilic or polar so that while there is a hydrophobic component, the nanoemulsion type remains oil in water $(\mathrm{o} / \mathrm{w})$. The results are in accordance with the desired because the type nanoemulsion o/w easily removed from the skin.

\section{Particle size and globule size distribution}

Measurements were made using PSA in the $1^{\text {st }}$ week. The particle measurement results show that each formula has a variable size, this is due to the difficulty of homogenizing two different systems, in addition to several other factors such as duration or speed of stirring also causes the formula is not homogeneous. The particle size from the $1^{\text {st }}$ week of the eighth increases, as most of the tween 80 undergoes hydrolysis, thus reducing the effectiveness of the globule nanoemulsion interface film layer [17]. Temperature modification during storage can cause a decrease in the effectiveness of the surfactant so that oil droplets tend to close together and eventually the droplets produce larger droplets. Globule size distribution is an important characteristic of the nanoemulsion system, as it may affect drug release and stability of the preparation [18].

The polydispersity index (PDI) is an indicator of the size distribution of particle in the emulsion, PDI value close to zero denotes the monodispersion system and PDI value close to 1.0 suggesting that the emulsion has a very broad size distribution. Therefore, PDI values lower than 0.2 indicate homogenous populations, while a 0.3 value represents heterogeneity. The desirable characteristics of the formulation are the high stability of two immiscible components to stay mixed as one phase emulsion. The acceptable PDI value should be $<0.7$ depending on the sample type [19].

The results of this study indicate that the PDI values for the F8 and F9 formulas are $>0.7$ which means that the two formulas have a broad particle size distribution (polydispersion) and include unstable formula.

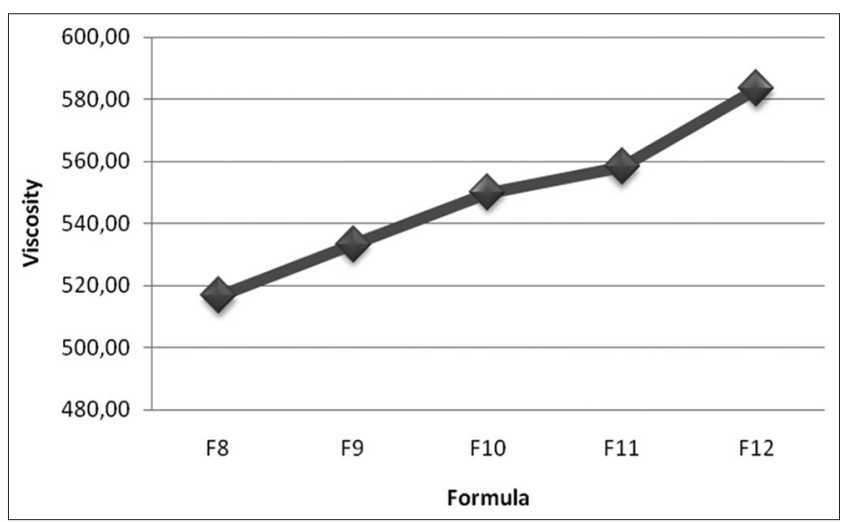

Fig. 1: Graph of viscosity measurement of formula

The PDI value of the formulae F10 to F12 is below 0.7 , so it belongs to the polydispersion category and is still included in the stable formula category. The measurement result of particle size and globule size distribution of nanoemulsion formula using PSA is shown in Table 5.

\section{Physical stability test}

Storage at a temperature of $4^{\circ} \pm 2^{\circ} \mathrm{C}$

All formulas are stable until the storage of the $5^{\text {th }}$ week, but in the $6^{\text {th }}$ week, the formulae F8 and F9 become unstable because at the bottom of the glass there are fine particles. This is due to the decreasing effectiveness of the surfactant so that the oil droplets tend to close together and eventually the united droplets produce larger droplets that settle on the bottom of the glass.

Storage at a temperature $25^{\circ} \pm 2^{\circ} \mathrm{C}$

All formulas are stable until the storage of the $5^{\text {th }}$ week, but in the $6^{\text {th }}$ week, the formulae F8 and F9 become unstable because at the bottom of the glass there are fine particles. This is because the surfactant has not been effective for dissolving ketoconazole and its effectiveness in the formula is also decreased so that the ketoconazole molecules tend to unite and that settle on the bottom of the glass [15]

Storage at a temperature $40^{\circ} \pm 2^{\circ} \mathrm{C}$

All formulas were stable in storage until week 7 , in the $8^{\text {th }}$ week with the formulae F11 and F12 unstable because the color changed to weak orange and the $\mathrm{pH}$ decreased. At high concentrations of tween 80 and increased temperatures, degradation rate increased due to the tween 80 autoxidation reaction $[17,20]$

Cycling test

All formulas are stable during storage.

\section{Centrifugation test (mechanical test)}

All formulas are stable and there is no phase separation, which means that all formulas are stable against the gravitational force experienced for 1 year. 
Table 5: The particle size and PDI values of the nanoemulsion formula

\begin{tabular}{lccc}
\hline Formula & Particle size & Intensity & PDI \\
\hline F8 & 43.05 & 0.93 & 1.08 \\
& 249.49 & 0.07 & \\
F9 & 38.91 & 0.93 & 1.01 \\
& 234.49 & 0.07 & \\
F10 & 29.34 & 0.88 & 0.62 \\
& 64.58 & 0.12 & \\
F11 & 3.09 & 0.06 & 0.68 \\
& 16.22 & 0.59 & \\
& 58.86 & 0.35 & 0.68 \\
F12 & 3.86 & 0.08 & \\
& 11.99 & 0.61 & \\
\end{tabular}

PDI: Polydispersity index

\section{CONCLUSIONS}

Optimization of surfactants, cosurfactants, and oils to form a stable ketoconazole nanoemulsion formulation by spontaneous emulsification method can be at surfactant concentrations (tween 80) $36 \%$, cosurfactant (ethanol) 9\%, and oil 5\% (IPM).

\section{ACKNOWLEDGMENTS}

The authors would like to thank Iksen, B.Pharm., M.Sc. (USU), for his neverending guidance and suggestions throughout the preparation of this article.

\section{AUTHOR'S CONTRIBUTIONS}

All the authors have contributed equally.

\section{CONFLICTS OF INTERESTS}

Declared none.

\section{REFERENCES}

1. Shankar R, Tiwari V, Mishra C, Sing CK, Sharma D, Jaiwal S. Formulation and evaluation of ketoconazole nanoemulsion gel for topical delivery. Am J PharmTech Res 2015;5:445-62.

2. Patel HC, Parmar G, Seth AK, Patel JD, Patel SR. Formulation and evaluation of o/w nanoemulsion of ketoconazole. Pharma Sci Monitor 2013;4:338-51.

3. Khatab A, Ismail S. Formulation and evaluation of oxiconazole nitrate mucoadhesive nanoemulsion based gel for treatment of fungal vaginal infection. Int J Pharm Pharm Sci 2016;8:34-40.
4. Chellapa P, Mohamed AT, Keleb EI, Elmahgoubi A, Eid AM, Issa YS, et al. Nanoemulsion and nanoemulgel as a topical formulation. IOSR J Pharm 2015;5:43-7.

5. Giongo JL, Vaucher RD, Ourique AF, Steffler MC, Frizzo CP, Hennemman B, et al. Development of nanoemulsion containing pelargonium graveolens oils: Characterization and stability study. Int J Pharm Pharm Sci 2016;5:271-6.

6. Lee KW, Omar D, Abdan K, Wong MY. Physicochemical characterization of nanoemulsion formulation of phenazine and their antifungal efficacy against ganoderma boninense PER71 in vitro. Res J Pharm Biol Chem Sci 2016;7:3056-66.

7. Chime SA, Kenechukwu FC, Attama AA. Application of Nanotechnology in Drug Delivery. United Kingdom: Intech; 2014.

8. Mishra RK, Soni GC, Mishra RP. A review article on nanoemulsion. Word J Pharm Pharm Sci 2014;3:258-74.

9. Costa SD, Basri M, Shamsudin N, Basri H. Stability of positively charged nanoemulsion formulation containing steroidal drug for effective transdermal application. J Chem 2014;2014:1-8.

10. Makhmalzadeh BS, Torabi S, Azarpanah A. Optimization of ibuprofen delivery through rat skin from traditional and novel nanoemulsion formulations. Iran J Pharm Res 2012;11:47-58.

11. Patel RR, Patel ZK, Patel KR, Patel MR. Formulation and evaluation of microemulsion based gel of ketoconazole. Int J Univ Pharma Biosci 2014;3:93-111

12. Derie DV, Burade KB, Kotwal RS, Galkwad VB. Formulation and evaluation of microemulsion based gel for topical delivery of ketoconazole. Indian Drugs 2008;45:138-40.

13. Jufri M, Natalia M. Physical stability and antibacterial activity of black cumin oil (Nigella sativa L.) nanoemulsion gel. Int J Pharmtech Res 2014;6:1162-9

14. Patil MP, Shinde GP, Kshirsagar SJ, Parakh DR. Development and characterization of ketoconazole loaded organogel for topical drug delivery. Inventi J 2015;3:1-10.

15. Fletcher J. Making the Connection-Particle Size, Size Distribution, and Rheology. Chemie.DE Information Service GmbH; 2012. Available from: http://www.chemeurope.com/en/whitepapers/61207/making-theconnection-particle-size-size-distribution-and-rheology.html.

16. Saberi AH, Fang Y, McClements DJ. Fabrication of vitamin E-enriched nanoemulsions: Factors affecting particle size using spontaneous emulsification. J Colloid Interface Sci 2013;391:95-102.

17. Kishore, Pappenberger A, Dauphin IB, Ross A, Buergi B, Staempfli A, et al. The degradation of polysorbates 20 and 80 and its potential impact on the stability of biotherapeutics. Pharm Res 2011;28: 1194-210.

18. Mohanraj VJ, Chen Y. Nanoparticles-a review. Trop J Pharm Res 2006;5:561-73.

19. Lee KW, Omar D, Abdan K, Wong MY. Physiochemical characterization of nanoemulsion formulation of phenazine and their antifungal efficacy against ganoderma boninense PER71 in vitro. Res J Pharm Biochem Sci 2016;7:3056-66.

20. Mhaske RA, Sahasrabudhe S. Identification of major degradation products of ketoconazole. Sci Pharm 2011;79:817-36. 